\title{
P11: Analyse von Gutachten des MDK zum Zeitaufwand für Hilfen bei den gesetzlich definierten Verrichtungen der Grundpflege
}

\author{
Jens Weidner
}

Online publiziert: 24. Oktober 2013

(C) Springer-Verlag Wien 2013

Zielstellung: In der Untersuchung wurden die Formulargutachten des MDK und freier Pflegesachverständiger hinsichtlich Diskrepanzen bei der zeitlichen Bemessung für Hilfen bei den Verrichtungen der Grundpflege analysiert.

Methodik: Der Zugang zur Population erfolgte über eine Rechtsanwaltskanzlei in Dresden. Voraussetzung waren Fälle aus den Jahren 2010 bis 2012, bei denen neben standardisierten Formulargutachten des MDK auch standardisierte Formulargutachten freier Pflegesachverständiger vorhanden waren. Es wurden 87 in die Datenauswertung mit SPSS 15 eingeschlossen. Neben deskriptiven Methoden kamen interferenzstatistische Testverfahren zur Anwendung. Es wurde ein Signifikanzniveau von $\alpha<5 \%$ festgelegt.

Ergebnisse: Es konnte festgestellt werden, dass die Diskrepanzen bei der zeitlichen Bemessung für Hilfen bei den gesetzlich definierten Verrichtungen signifikant sind. Zugleich zeigt die Untersuchung, dass die zeitlichen Bemessungen für die Verrichtungen der Grundpflege eine hohe Streuung aufweisen, was auf heterogene Pflegehandlungen schließen lässt.
Diskussion: Untersucht wurden Unterschiede hinsichtlich der Zeitbedarfe der Grundpflege zwischen den gutachterlichen Feststellungen des MDK und den Feststellungen der freien Pflegesachverständigen. Es handelte sich um eine Vollerhebung. Die untersuchten Daten weisen eine hohe Güte auf, da beide Gutachterparteien im Rahmen der Feststellung über das Vorliegen von Pflegebedürftigkeit tätig wurden und die Begutachtungen standardisiert sind. Für die Mandantschaft der Rechtsanwaltskanzlei sind die Ergebnisse repräsentativ. Kritisch zu betrachten ist die Tatsache, dass die freien Pflegesachverständigen im Rahmen des widersprochenen Verwaltungsaktes zum Einsatz kamen.

Schlussfolgerung: Deutlich wurde, dass die zeitliche Bemessung des Hilfebedarfs zwischen den Gutachterparteien signifikante Diskrepanzen aufweist. Die festgestellte Streuung der einzelnen Zeitbedarfe deutet daraufhin, dass die Bemessung der Hilfen sowohl bei den Gutachtern des MDK als auch bei den freien Pflegesachverständigen unterschiedlich ist. Das lässt den Schluss zu, dass die Pflegehandlungen bei den einzelnen Verrichtungen heterogen sind.

J. Weidner $(\triangle)$

Studienzentrum Dresden, Fachbereich Gesundheit und Pflege,

Hamburger Fernhochschule, Dresden, Deutschland

E-Mail: jens.weidner@campus.hamburger-fh.de 